\title{
Infrared study of the magnetostructural phase transition in correlated $\mathrm{CrN}$
}

\author{
J. Ebad-Allah, ${ }^{1,2}$ B. Kugelmann, ${ }^{1}$ F. Rivadulla ${ }^{3}$ and C. A. Kuntscher ${ }^{1, *}$ \\ ${ }^{1}$ Experimentalphysik 2, Universität Augsburg, D-86135 Augsburg, Germany \\ ${ }^{2}$ Department of Physics, Tanta University, 31527 Tanta, Egypt \\ ${ }^{3}$ Centro de Investigación en Química Biolóxica e Materiais Moleculares (CIQUS) \\ and Departamento de Química-Física, Universidade de Santiago de Compostela, 15782 Santiago de Compostela, Spain
}

(Received 26 April 2016; revised manuscript received 23 August 2016; published 9 November 2016)

\begin{abstract}
We report on the pressure and temperature dependence of the electronic and vibrational properties of polycrystalline $\mathrm{CrN}$ studied by optical transmission and reflection measurements over the frequency range 0.012-2.48 eV. The optical conductivity spectrum of $\mathrm{CrN}$ at ambient conditions shows a phonon mode at $\approx 55 \mathrm{meV}$ with a shoulder at $\approx 69 \mathrm{meV}$, a pronounced midinfrared absorption band centered at $123 \pm 2 \mathrm{meV}$, and a high-energy absorption band at $\approx 1.5 \mathrm{eV}$. The absorption bands are discussed in terms of the charge-transfer insulator picture. Following the reflectance spectrum with increasing pressure, the activation of an additional phonon mode above 0.6 GPa indicates the occurrence of a pressure-induced structural phase transition. Furthermore, the absorption spectrum exhibits significant changes in the far-infrared range with decreasing temperature: The phonon mode shows a sudden broadening followed by a splitting below $270 \mathrm{~K}$. These changes observed under pressure or while cooling down can be associated with the magnetostructural phase transition reported previously.
\end{abstract}

DOI: 10.1103/PhysRevB.94.195118

\section{INTRODUCTION}

Transition-metal nitrides provide remarkable properties such as high hardness, mechanical strength, wear and corrosion resistance, high-temperature oxidation resistance, and good chemical as well as thermal stability [1-3]. Furthermore, they have been used in semiconductor devices because of their relatively high electrical conductivity [1-4]. Among the nitrides, $\mathrm{CrN}$ is the only compound which does not have a ground state of cubic symmetry, making it especially interesting. At ambient conditions, $\mathrm{CrN}$ is in a paramagnetic phase with a cubic rock-salt structure similar to other transition-metal nitrides. At the Néel temperature $T_{N}$ with values in the range 270-286 $\mathrm{K}$ reported in the literature [5-8], the material undergoes a magnetic phase transition to an ordered antiferromagnetic phase. The magnetic phase transition is accompanied by a structural phase transition from the cubic phase to an orthorhombic phase. Recently, it has been reported [9] that this magnetostructural phase transition could also be induced by applying pressure of $\approx 1 \mathrm{GPa}$ at room temperature. The pressure-induced phase transition at $\approx 1 \mathrm{GPa}$ was found to be accompanied by a reduction of the bulk modulus of about $25 \%$ and a $0.56-0.59 \%$ increase in the atomic density [9].

The electrical transport studies of $\mathrm{CrN}$ revealed discrepant results regarding the temperature dependence of the resistivity. Several transport studies on polycrystalline samples [10-12] and thin films grown on $\mathrm{MgO}$ (001) substrates [13] supported the semiconductor scenario in the whole temperature range below $400 \mathrm{~K}$, indicating the existence of a band gap. In contrast, Constantin et al. observed a change from semiconductor to metallic behavior in the conductivity spectrum at $T_{N}$ on a thin film of $\mathrm{CrN}$, while the recent study of Quintela et al. [14] on a thin film of $\mathrm{CrN}$ reported a metallic-like behavior for the

\footnotetext{
*christine.kuntscher@physik.uni-augsburg.de
}

annealed $\mathrm{CrN}$ thin film in the temperature range below $400 \mathrm{~K}$, with a steep decrease of the resistivity at $T_{N}$ associated with the magnetostructural phase transition. A metallic behavior was also observed in other resistivity studies in the temperature range below $300 \mathrm{~K}[5,15]$.

Theoretical works interpreted the observed magnetic, structural, and electronic properties of $\mathrm{CrN}$ in terms of charge ordering or Mott-insulating behavior, which is characteristic for correlated electron systems [16]. Theoretical calculations using the local spin-density approximation corrected by Hubbard Coulomb terms for the $d$ electrons (LSDA+U) [17] supported the idea that $\mathrm{CrN}$ is close to a charge-transfer type insulator. Here, a spin separation of states near the Fermi level was reported, leading to opening up a small charge gap of less than $1 \mathrm{eV}$ between the $\mathrm{N} 2 p$-type bands and $\mathrm{Cr} 3 d$-type bands. This small energy gap was suggested to be related to the measured optical charge gap of $0.64 \mathrm{eV}$ extracted from the absorbance spectrum $[13,18]$. In contrast, the analysis of the dielectric constant determined from transmission and reflection measurements in broad frequency ranges $(0.04-5 \mathrm{eV}$ and $0.1-5 \mathrm{eV}$, respectively) indicated the presence of a small indirect band gap of $0.19 \pm$ $0.46 \mathrm{eV}$ at the Fermi level. This gap was attributed to the formation of local magnetic moments and electron interaction effects, suggesting that $\mathrm{CrN}$ is a Mott-Hubbard-type insulator [19].

Here, we report on the electronic and vibrational properties of polycrystalline $\mathrm{CrN}$ investigated by reflectance and transmission measurements in a broad frequency range 0.012 $2.48 \mathrm{eV}\left(100-20000 \mathrm{~cm}^{-1}\right)$. The goal of our optical study was to verify the semiconducting character suggested by electronic transport measurements, with an energy gap smaller than $150 \mathrm{meV}$ at ambient conditions [13,18-20]. Furthermore, absorption measurements for temperatures between 300 and $150 \mathrm{~K}$ as well as room-temperature reflectance measurements under high pressure up to $4.5 \mathrm{GPa}$ were carried out to search for 
signatures of the expected temperature- and pressure-induced magnetostructural transition.

\section{EXPERIMENT}

The CrN powder was synthesized by ammonolysis of $\mathrm{Cr}_{3} \mathrm{~S}_{4}$ at $800{ }^{\circ} \mathrm{C}$ for $10 \mathrm{~h}$; details on the powder preparation are reported in Refs. [10,11].

For the temperature-dependent transmittance studies, the $\mathrm{CrN}$ powder was mixed with $\mathrm{CsI}$ in a certain ratio; here we used $0.14 \%$ of $\mathrm{CrN}$ to prepare the $\mathrm{CrN} / \mathrm{CsI}$ pellet. The mixture was pressed to a sintered pellet of about $50 \mu \mathrm{m}$ thick. Then pellets of a mixture of CrN/CsI and of pure CsI were mounted on the holder of a cold-finger cryostat (Cryo Vac Konti-Mikro cryostat). CsI was chosen due to its transparent in the infrared frequency range. The actual temperature of the sample was measured by a sensor attached in direct vicinity of the pellets. We measured the intensity of the radiation transmitted by the $\mathrm{CrN}$ pellet, $I_{s}(\omega)$; as reference, the intensity $I_{r}(\omega)$ transmitted by a pure CsI pellet was used. The ratio $T=I_{s}(\omega) / I_{r}(\omega)$ is a measure of the transmittance of the sample, and the corresponding absorbance was calculated according to $A=\log _{10}(1 / T)$.

The reflectance measurement at ambient conditions was conducted in the frequency range 0.012-2.48 eV (100-20 000 $\mathrm{cm}^{-1}$ ) using a Bruker IFS 66v/S Fourier transform infrared (FT-IR) spectrometer with an infrared microscope (Bruker IR scope II) equipped with a $15 \times$ magnification objective. We measured the intensity of the radiation reflected from a pure $\mathrm{CrN}$ pellet, $I_{S}(\omega)$; as reference we measured the intensity reflected from an Ag mirror, $I_{r}(\omega)$. The ratio $R=I_{s}(\omega) / I_{r}(\omega)$ is a measure of the reflectance of the sample. The real part of the optical conductivity $\sigma_{1}$ was obtained via Kramers Kronig (KK) transformation. Hereby, the reflectivity data were extrapolated to low frequencies based on a Lorentz fit. For the high-frequency extrapolation we used the $\mathrm{x}$-ray atomic scattering functions [21]. Alternatively, we measured the UV range up to around $5 \mathrm{eV}$, then used the power law of $(1 / \omega)$ to extrapolate the reflectivity data. By this way we estimated the error bars and found that the high-energy extrapolation affects the high-energy features of the obtained optical conductivity only slightly.

Pressure-dependent reflectance experiments were carried out at room temperature in the far-infrared frequency range. For the generation of pressures up to $4.5 \mathrm{GPa}$, a clamp diamond anvil cell (Diacell cryoDAC-Mega) equipped with type IIA diamonds, which are suitable for infrared measurements, was used. A small piece (lateral dimensions of about $160 \mu \mathrm{m} \times$ $150 \mu \mathrm{m}$, with a thickness of $\sim 50 \mu \mathrm{m}$ ) was cut from a pure $\mathrm{CrN}$ pellet and placed in the hole of a $\mathrm{CuBe}$ gasket. Finely ground CsI powder served as quasi-hydrostatic pressure transmitting medium. The pressure in the diamond anvil cell was determined in situ by the ruby luminescence method [22]. As reference, we used the intensity reflected from the $\mathrm{CuBe}$ gasket inside the DAC. All the pressure-dependent reflectance spectra refer to the absolute reflectance at the sample-diamond interface, denoted as $R_{s-d}$. Further information about the geometry of the reflectivity measurements can be found in our earlier publications $[23,24]$.

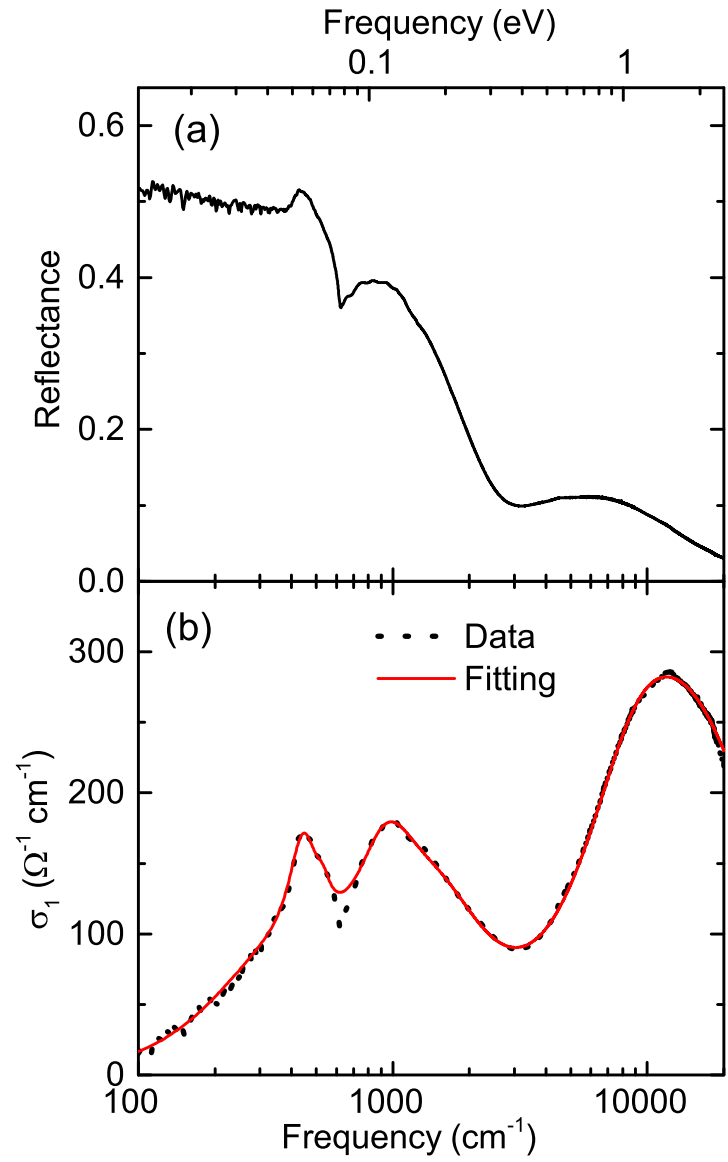

FIG. 1. (a) Reflectance of a pure $\mathrm{CrN}$ pellet over a broad frequency range at ambient conditions, used for the $\mathrm{KK}$ analysis to extract the optical conductivity. (b) Lorentz model fit of the corresponding real part $\sigma_{1}$ of the optical conductivity, obtained by $\mathrm{KK}$ analysis, at ambient conditions.

\section{RESULTS AND DISCUSSION}

\section{A. Optical spectrum at ambient conditions}

The ambient-condition reflectance spectrum of the pure $\mathrm{CrN}$ pellet over a broad frequency range and the corresponding real part $\sigma_{1}$ of the optical conductivity are depicted in Fig. 1. The reflectance spectrum consists of several contributions: a broad peak with a shoulder in the far-infrared range followed by a pronounced midinfrared (MIR) absorption band and a high-energy absorption band. For a quantitative analysis of the observed features, we fitted the optical conductivity $\sigma_{1}$ with the Lorentz model. The far-infrared peak and its shoulder were described by two Lorentzian functions. Furthermore, both the MIR band and the high-energy band were described by two Lorentzian functions each. Figure 1(b) shows the fit of the $\sigma_{1}$ spectrum with the Lorentz model. The finite conductivity in the far-infrared range does not give a clear indication of a Drudetype contribution, supporting a semiconducting behavior of CrN above $T_{N}[10-13,25]$.

From the fitting we obtained the frequency position of the far-infrared peak at $\approx 55 \mathrm{meV}$. This peak is related to the transverse-optical (TO) mode predicted at $48.7 \pm$ $0.2 \mathrm{meV}$ [19]. The shoulder observed at around $69 \mathrm{meV}$ could 
be attributed to the predicted longitudinal optical mode located at $75.0 \pm 6.8 \mathrm{meV}$ [19]. Our results are slightly different from the previously published reflectance data, which show a broad phonon mode at around $52 \mathrm{meV}$ [19]. The small difference in the position of the phonon mode could be attributed to the thickness, the composition, and the strain of the thin films used in the previous measurements, which affect the phonon mode position $[20,26]$.

The MIR band located at $123 \pm 2 \mathrm{meV}$ can be attributed to a charge gap of size $\Delta \approx 100-150 \mathrm{meV}$, consistent with the activated behavior observed by several resistivity studies which found activation energy $E_{a}$ values in the range 50$75 \mathrm{meV}$ [10-13,25]. Furthermore, we observe a high-energy band located at around $1.5 \mathrm{eV}$, in good agreement with the electronic interband transitions occurring at around $1.5 \mathrm{eV}$ as observed by Zhang et al. [19].

In order to attribute the observed absorption bands to specific optical excitations, we will refer to the band-structure calculations of Herwadkar et al. using the LSDA+U approximation [17]. Here, for the cubic structure a strong depletion in the density of states by the spin separation of states near the Fermi level was predicted. A small gap between the N $2 p$-type bands and $\mathrm{Cr} 3 d$-type bands was found, suggesting that $\mathrm{CrN}$ is likely to be a charge-transfer-type insulator [16]. The size of the smallest energy gap was predicted to depend on the value of the onsite Coulomb repulsion $U$ and the type of the magnetic configurations of the cubic phase: either ferromagnetic (FM) phase or one of the distorted antiferromagnetic (AFM) phases $[110]_{1}$ and $[110]_{2}$. As an example, for $U=1 \mathrm{eV}$ the band structure of the AFM-[110] 2 phase showed the removal of some bands at the $\Gamma$ point from the region near $E_{F}$, leading to the opening of an indirect gap smaller than 0.1 $\mathrm{eV}$. When increasing $U$ to $3 \mathrm{eV}$, both of the AFM-[110] 2 and FM phases' band structures showed the opening of a small direct gap slightly less than $1 \mathrm{eV}$ near the $\Gamma$ point, while for the AFM-[110] $]_{1}$ phase the gap was found to be about $0.4 \mathrm{eV}$. This small gap was suggested to be opened between the $\mathrm{N}$ $2 p$-type valence band and the majority-spin $e_{g}$ bands of $\mathrm{Cr}$, and hence $\mathrm{CrN}$ was interpreted in terms of a charge-transfer type insulator, even for small values of $U$. According to the calculations of Herwadkar et al., the MIR band observed in our optical data at around $125 \mathrm{meV}$ can be explained in terms of a charge transfer $\Delta$ from the $\mathrm{N} 2 p$-type bands to the $\mathrm{Cr} 3 d$-type bands. This would support the transport data, which proposed that $\mathrm{CrN}$ is located in the charge transfer insulator regime, close to the itinerant electron limit [10,11]. Furthermore, the band structure calculations [17] showed another energy gap at around $1.8 \mathrm{eV}$, opened between the minority spin N $2 p$-type valence band and the minority-spin $t_{2 g}$ band at the $X$ point. This could explain the high-energy band at around $1.5 \mathrm{eV}$ observed in our data and the data of Zhang et al. Accordingly, we suggest that both the MIR band and the high-energy band could be explained in terms of a charge-transfer insulator rather than a Mott insulator. A charge gap of similar size was also observed for other $3 d$ transition-metal compounds, like $\mathrm{Ni}_{1-\delta} \mathrm{S}, \mathrm{BaTiO}_{3}$ and the family of the rare-earth $(R)$ transition metal oxides $\left(R M \mathrm{O}_{3}\right.$, with $M=\mathrm{Sc}, \mathrm{Ti}, \mathrm{V}, \mathrm{Cr}, \mathrm{Mn}, \mathrm{Fe}, \mathrm{Co}, \mathrm{Ni}$, and $\left.\mathrm{Cu}\right)$, and interpreted in terms of a charge transfer between $2 p$-type and $3 d$-type states [27-29].

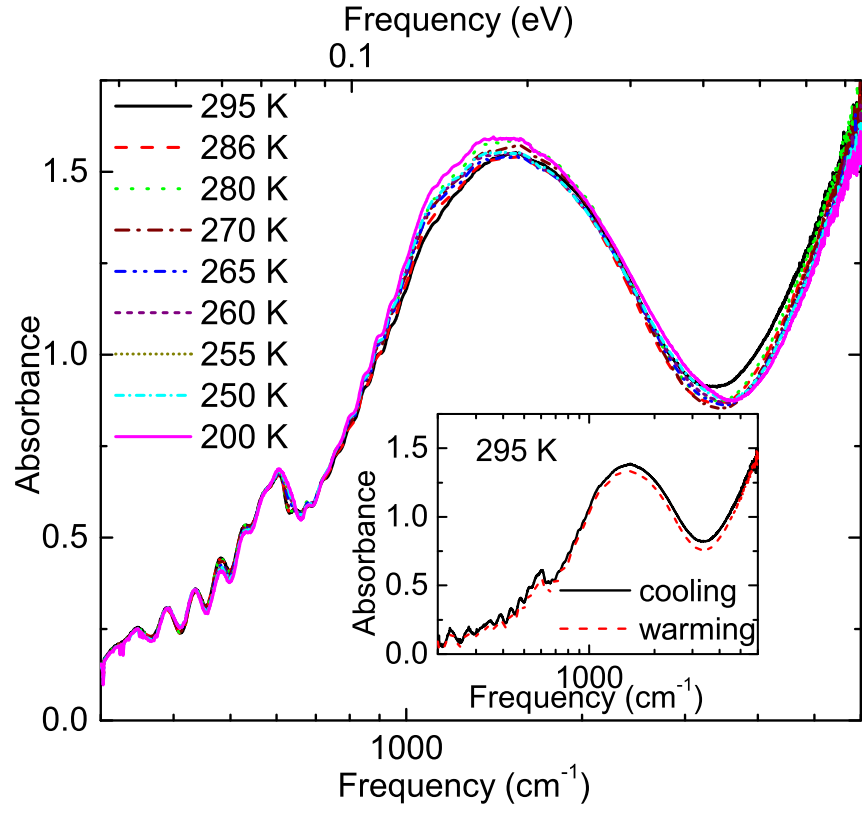

FIG. 2. Temperature-dependent absorbance of a polycrystalline $\mathrm{CrN} / \mathrm{CsI}$ pellet at ambient pressure. Inset shows the absorbance spectra at room temperature before cooling down and after warming up.

\section{B. Temperature-dependent optical spectra}

Ambient-pressure absorbance spectra of $\mathrm{CrN}$ for several selected temperatures are displayed in Fig. 2 in the frequency range $0.025-0.868 \mathrm{eV}\left(200-7000 \mathrm{~cm}^{-1}\right)$. The wiggles in the spectra are due to Fabry-Perot interferences due to multiple reflections within the sample. As the temperature is lowered, the absorption spectra do not show significant spectral changes below $0.062 \mathrm{eV}\left(500 \mathrm{~cm}^{-1}\right)$, consistent with the electrical transport measurements which found an activated behavior for $\mathrm{CrN}$ for the temperature range below $400 \mathrm{~K}[10,11]$. Additionally, the MIR absorption band shows only a slight shift to lower frequencies during cooling down, indicating that the temperature effect on the activation energy is very small. The major changes were observed in the phonon mode range, i.e., between $0.068 \mathrm{eV}\left(550 \mathrm{~cm}^{-1}\right)$ and $0.083 \mathrm{eV}\left(670 \mathrm{~cm}^{-1}\right)$. With decreasing temperature, the phonon mode shows a sudden broadening below $270 \mathrm{~K}$, followed by a splitting during further cooling down, as depicted in Fig. 3(a). The changes in the phonon mode with temperature are illustrated by the normalized difference $\Delta A$, defined as

$$
\Delta A(\omega, T)=[A(\omega, T)-A(\omega, 300 \mathrm{~K})] / A(\omega, 300 \mathrm{~K}) .
$$

The temperature-dependent absorbance spectra and the corresponding normalized difference spectra $\Delta A(\omega, T)$ are shown in Fig. 3 in the upper and lower panels, respectively. With decreasing temperature, $\triangle A(\omega, T)$ has a slope of around zero for $T>280 \mathrm{~K}$, while below $280 \mathrm{~K}$ the slope starts to change in the frequency range $0.068-0.083 \mathrm{eV}(550$ $\left.670 \mathrm{~cm}^{-1}\right)$. In particular, Fig. 3(c) shows that $\Delta A(\omega, T)$ clearly increases below $270 \mathrm{~K}$ during cooling, indicating the splitting of the phonon mode.

The broadening and splitting of the phonon mode at $T_{N}$ are consistent with a symmetry lowering of the crystal structure 


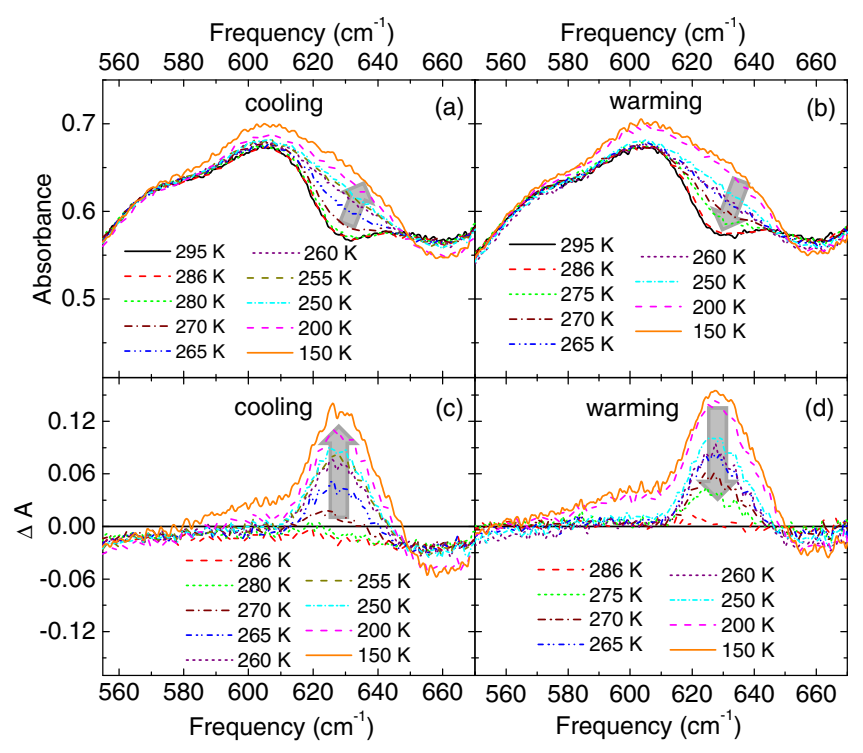

FIG. 3. The two panels (a) and (b) depict the absorbance of a $\mathrm{CrN} / \mathrm{CsI}$ pellet as a function of temperature. The two panels (c) and (d) show the normalized difference $\Delta A(\omega, T)$ in the far infrared range, illustrating the evolution of the phonon mode as a function of temperature above and below the phase transition temperature. Block arrows illustrate the changes during cooling and warming.

from a cubic rock-salt structure with space group $(F m 3 m)$ to an orthorhombic phase with space group (Pnma) [6,7,30]. These structural changes are accompanied by a magnetic phase transition from a paramagnetic phase to an ordered antiferromagnetic phase at $T_{N}$ as shown by several studies [5-8].

During warming up, $\triangle A(\omega, T)$ is basically flat above $275 \mathrm{~K}$ in the frequency range $0.068-0.083 \mathrm{eV}\left(550-670 \mathrm{~cm}^{-1}\right)$ [see Fig. 3(d)], indicating the absence of any broadening or splitting of the phonon mode. The width of the so-obtained thermal hysteresis amounts to $\approx 5 \mathrm{~K}$. The thermal hysteresis was observed in earlier studies, but with different values of the width depending on the $\mathrm{N}$ concentration [10-13,25]. Browne et al. [5] reported a hysteresis with a width of $\approx 3 \mathrm{~K}$, which is close to our result, while Constantine et al. [25] found a higher value of $\approx 20 \mathrm{~K}$. The thermal hysteresis indicates that the phase transition is of first order, as proposed earlier [5,25]. Furthermore, we note that the observed temperature-induced changes are reversible (see the inset of Fig. 2).

\section{Pressure-dependent optical spectra}

The room-temperature reflectance spectra of $\mathrm{CrN}$ for selected pressures between 0.2 and $4.5 \mathrm{GPa}$ in the frequency range $0.02-0.086 \mathrm{eV}\left(180-700 \mathrm{~cm}^{-1}\right)$ are depicted in Fig. 4. At the lowest pressure $(\approx 0.2 \mathrm{GPa})$ a broad phonon mode and a shoulder are observed, consistent with our data at ambient conditions discussed above. The overall reflectance increases monotonically with increasing pressure, which might indicate a growth of spectral weight within the measured frequency range. However, we cannot exclude that the overall increase is due to pressure-induced changes in the surface flatness, since the CrN pellet is pressed on the diamond anvil. This

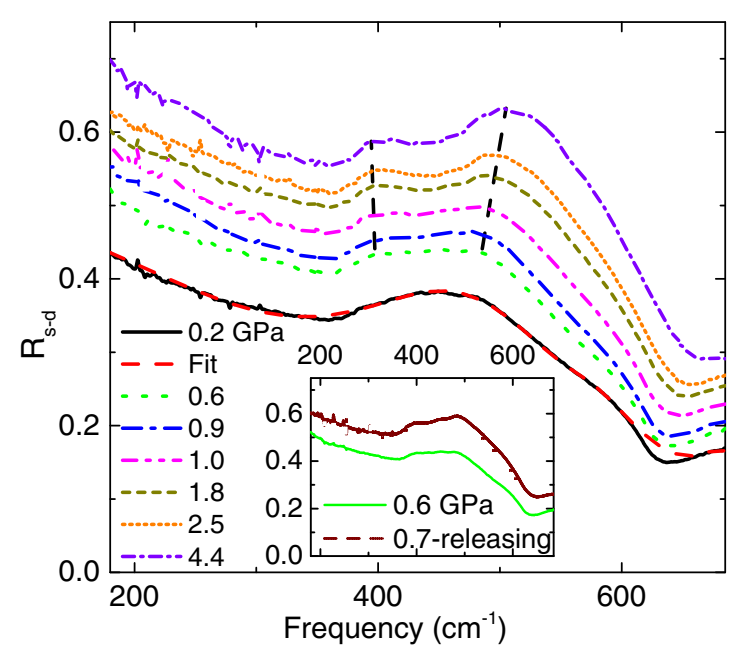

FIG. 4. Pressure-dependent reflectance of a pure $\mathrm{CrN}$ pellet at room temperature in the low-frequency range for several selected pressures between 0.2 and $4.4 \mathrm{GPa}$. The dashed red line is the fit of the reflectance spectrum at $0.2 \mathrm{GPa}$ using the Lorentz model. The two dashed black lines illustrate the splitting of the phonon mode.

might also explain the rather high overall reflectance after pressure release at $0.7 \mathrm{GPa}$ (see inset of Fig. 4). Nevertheless, the nonreversibility of the pressure-induced effects cannot be excluded as a possible reason for the discrepancy between the spectra at around 0.6 GPa during pressure increase and after pressure release.

The pressure-dependent phonon spectrum was analyzed by fitting with the Lorentz model. Hereby, the reflectance spectra were fitted according to the Fresnel equation for normalincidence reflectivity taking into account the diamond-sample interface $R_{s-d}$. Further information about Fresnel equation for $R_{s-d}$ is included in an earlier publication [31]. The phonon modes are described by two Lorentz functions. As an example, we show in Fig. 4 the fit of the reflectance spectrum at $0.2 \mathrm{GPa}$. Above $0.6 \mathrm{GPa}$ an activation of a phonon mode at $\approx 50 \mathrm{meV}$ occurs, which is directly seen in the reflectance spectra (see the dashed lines in Fig. 4). Therefore, an additional Lorentz term had to be included in the fit.

Based on the Lorentz fitting, the phonon frequencies were extracted and are plotted in Fig. 5 as a function of pressure. With increasing pressure, the phonon mode at $\approx 55$ meV slightly shifts to higher energy, while the shoulder at $\approx 69 \mathrm{meV}$ broadens. Furthermore, the activation of a phonon mode located at around $50 \mathrm{meV}$ indicates a pressure-induced symmetry lowering of the crystal structure above $0.6 \mathrm{GPa}$. This finding supports the structural phase transition observed by Rivadulla et al. [9], from the cubic rock salt to an orthorhombic crystal structure at $\approx 1 \mathrm{GPa}$. Concomitant with the structural phase transition, a large reduction of the bulk modulus $K_{0}$ from 340(10) GPa for the cubic rock-salt phase to 243(10) GPa for the orthorhombic phase was reported; i.e., $\mathrm{CrN}$ softens under pressure.

\section{SUMMARY}

In summary, the electronic and vibrational properties of polycrystalline $\mathrm{CrN}$ have been investigated by transmission 


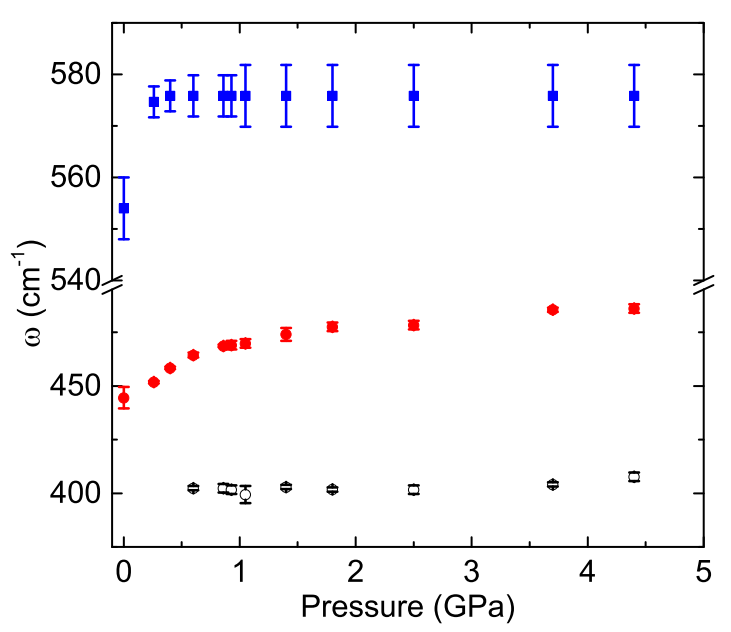

FIG. 5. Phonon frequencies as a function of pressure, obtained by fitting the reflectance spectra with the Lorentz model.

and reflection measurements in the infrared frequency range for temperatures between 300 and $150 \mathrm{~K}$, and under high pressure up to $4.5 \mathrm{GPa}$. At ambient conditions, the optical conductivity spectrum shows an optical phonon mode at $\approx 55 \mathrm{meV}$ with a shoulder at $\approx 69 \mathrm{meV}$, a MIR absorption band at $123 \pm 2 \mathrm{meV}$, and a high-energy absorption band at $\approx 1.5 \mathrm{eV}$. Both absorption bands can be related to charge transfer excitations, suggesting that $\mathrm{CrN}$ is a charge-transfertype insulator. While cooling down, the phonon mode exhibits a sudden broadening and a splitting below $T_{N}$, which confirms the occurrence of the expected magnetostructural phase transition. This magnetostructural phase transition seems to occur also under pressure according to our pressure-dependent reflectance measurements, revealing the activation of a phonon mode at $0.6 \mathrm{GPa}$, in agreement with earlier studies.

\section{ACKNOWLEDGMENTS}

We gratefully acknowledge the financial support from the Federal Ministry of Education and Research (BMBF), Germany, through Grant No. 05K13WA1 (Verbundprojekt 05K2013, Teilprojekt 1, PT-DESY), and the MINECO-spain through Project No. MAR2013-44673-R.
[1] U. Wiklund, M. Bromark, M. Larsson, P. Hedenqist, and S. Hogmark, Surf. Coat. Technol. 91, 57 (1997).

[2] C. Nouveau, M. A. Djouadi, O. Banakh, R. Sanjines, and F. Levy, Thin Solid Films 398-399, 490 (2001).

[3] A. Neckel, Int. J. Quantum Chem. 23, 1317 (1983).

[4] B. R. Zhao, L. Chen, H. L. Luo, M. D. Jack, and D. P. Mullin, Phys. Rev. B 29, 6198 (1984).

[5] J. D. Browne, P. R. Liddell, R. Street, and T. Mills, Phys. Status Solidi A 1, 715 (1970).

[6] L. M. Corliss, N. Elliott, and J. M. Hastings, Phys. Rev. 117, 929 (1960).

[7] R. M. Ibberson and R. Cywinski, Physica B (Amsterdam, Neth.) 180-181, 329 (1992).

[8] M. N. Eddine, F. Sayetat, and E. F. Bertaut, C. R. Seances Acad. Sci., Ser. B 269, 574 (1969).

[9] F. Rivadulla, M. Banobre-Lopez, C. X. Quintela, A. Pineiro, V. Pardo, D. Baldomir, M. A. C. R. Lopez-Quintela, J. Rivas, C. A. Ramos, H. Salva, J. S. Zhou, and J. B. Goodennough, Nat. Mater. 8, 947 (2009).

[10] C. X. Quintela, F. Rivadula, and J. Rivas, Appl. Phys. Lett. 94, 152103 (2009).

[11] C. X. Quintela, J. P. Podkaminer, M. N. Luckyanova, T. R. Paudel, E. L. Thies, D. A. Hillsberry, D. A. Tenne, E. Y. Tsymbal, G. Chen, C. Eom, and F. Rivadula, Adv. Mater. 27, 3032 (2015).

[12] P. S. Herle, M. S. Hedge, N. Y. Vasathacharya, S. Philip, M. V. R. Rao, and T. Sripathi, J. Solid State Chem. 134, 120 (1997).

[13] D. Gall, C.-S. Shin, R. T. Haasch, I. Petrov, and J. E. Greene, J. Appl. Phys. 91, 5882 (2002).

[14] C. X. Quintela, F. Rivadulla, and J. Rivas, Phys. Rev. B 82, 245201 (2010).

[15] K. Inumaru, K. Koyama, N. Imo-oka, and S. Yamanaka, Phys. Rev. B 75, 054416 (2007).

[16] M. Imada, A. Fujimori, and Y. Tokura, Rev. Mod. Phys. 70, 1039 (1998).
[17] A. Herwadkar and W. R. L. Lambrecht, Phys. Rev. B 79, 035125 (2009).

[18] P. A. Anderson, R. J. Kinsey, S. M. Durbin, A. Markwitz, V. J. Kennedy, A. Asavod, W. Gao, and R. J. Reeves, J. Appl. Phys. 98, 043903 (2005).

[19] X. Y. Zhang and D. Gall, Phys. Rev. B 82, 045116 (2010).

[20] M. Hirai, Y. Ueno, T. Suzuki, W. Jiang, C. Grigoriu, and K. Yatsuil, Jpn. J. Appl. Phys. 40, 1052 (2001).

[21] D. B. Tanner, Phys. Rev. B 91, 035123 (2015).

[22] K. Syassen, High Press. Res. 28, 75 (2008).

[23] C. A. Kuntscher, S. Frank, A. Pashkin, M. Hoinkis, M. Klemm, M. Sing, S. Horn, and R. Claessen, Phys. Rev. B 74, 184402 (2006).

[24] J. Ebad-Allah, L. Baldassarre, M. Sing, R. Claessen, V. A. M. Brabers, and C. A. Kuntscher, J. Phys. Condens. Matter 25, 035602 (2013).

[25] C. Constantin, M. B. Haider, D. Ingram, and A. R. Smith, J. Appl. Phys. Lett. 85, 6371 (2004).

[26] J. Shirahata, T. Ohori, H. Asami, T. Suzuki, T. Nakayama, H. Sematsu, and K. Niihara, Jpn. J. Appl. Phys. 50, $01 B E 03$ (2011).

[27] Ph. Ghosez, X. Gonze, Ph. Lambin, and J. P. Michenaud, Phys. Rev. B 51, 6765 (1995).

[28] T. Arima, Y. Tokura, and J. B. Torrance, Phys. Rev. B 48, 17006 (1993).

[29] H. Okamura, J. Naitoh, T. Nanba, M. Matoba, M. Nishioka, S. Anzai, I. Shimoyama, K. Fukui, H. Miura, H. Nakagawa, K. Nakagawa, and T. Kinoshita, Sol. Stat. Commun. 112, 91 (1999).

[30] P. Hones, N. Martin, M. Regula, and F. Levy, J. Appl. Phys. 36, 1023 (2003).

[31] J. Ebad-Allah, L. Baldassarre, M. Sing, R. Claessen, V. A. M. Brabers, and C. A. Kuntscher, J. Appl. Phys. 112, 073524 (2013). 